\title{
Use of Anakinra in Hospitalized Patients with Crystal-associated Arthritis
}

\author{
Jean W. Liew and Gregory C. Gardner
}

ABSTRACT. Objective. In this retrospective observational study, we assess the efficacy and safety of the interleukin 1 receptor antagonist anakinra in medically complex, hospitalized patients with acute gout and calcium pyrophosphate crystal arthritis.

Methods. Adult inpatients treated with anakinra from 2014 to 2017 were identified for inclusion. Charts were reviewed for demographics, comorbidities, laboratory data, pain scores, joint involvement, prior treatment, dosing and response to anakinra, concurrent infections, and surgical interventions. Response to anakinra treatment was determined from review of provider documentation, as well as recorded pain scores on a numeric scale.

Results. We identified 100 individuals accounting for 115 episodes of arthritis. This population was $82 \%$ male, with an average age of 60 years. Comorbidities included renal disease (45\%) and history of organ transplantation (14\%). Twenty-nine episodes of arthritis occurred in the perioperative setting. Concurrent infection was present in 34 episodes. Eighty-six episodes of arthritis had partial or complete response to anakinra within 4 days of treatment initiation; 66 episodes had partial or complete response within 1 day of anakinra administration. Anakinra was well tolerated.

Conclusion. To our knowledge, this is the largest observational study of anakinra use in the inpatient setting for the acute treatment of crystal-associated arthritis. We observed a rapid response to anakinra, with $75 \%$ of episodes significantly improving or completely resolving within 4 days of the first dose. Our data also support the use of this biologic agent in individuals with infections, as well as perioperative individuals and immunosuppressed transplant recipients. (First Release April 15 2019; J Rheumatol 2019;46:1345-9; doi:10.3899/jrheum.181018)

Key Indexing Terms:

GOUT

CALCIUM PYROPHOSPHATE

PSEUDOGOUT

ANAKINRA CRYSTAL-ASSOCIATED ARTHRITIS

Gout is an inflammatory condition in which the precipitation of monosodium urate (MSU) crystals within joints results in recurrent attacks of arthritis. Calcium pyrophosphate (CPP) crystal arthritis, also known as pseudogout, is a similar condition that more commonly affects older individuals or those with certain metabolic disorders such as hemochromatosis.

The prevalence of gout in the United States is estimated to be $3.9 \%{ }^{1}$. Comorbidities are common and in 1 study, the prevalence of hypertension was $74 \%$, chronic kidney disease (CKD) $53 \%$, diabetes mellitus 26\%, and congestive heart failure $11 \%^{2}$. Gout is independently associated with higher medical comorbidity and increases in both primary care and inpatient treatment ${ }^{3,4}$.

Both gout and CPP crystal arthritis are chronic diseases marked by acute arthritic attacks. The pharmacologic

From the Division of Rheumatology, Department of Medicine, University of Washington, Seattle, Washington, USA.

J.W. Liew, MD, Division of Rheumatology, Department of Medicine, University of Washington; G.C. Gardner, MD, Division of Rheumatology, Department of Medicine, University of Washington.

Address correspondence to Dr. J.W. Liew, University of Washington, 1959 NE Pacific St., BB561, Seattle, Washington 98195, USA.

E-mail:jwliew@uw.edu

Accepted for publication January 8, 2018. management of both conditions is similar, and per the most recent American College of Rheumatology guidelines, includes nonsteroidal antiinflammatory drugs (NSAID) and colchicine as first-line agents. In monoarticular flares, an intraarticular glucocorticoid (GC) injection may be administered. Polyarticular flares may be treated with systemic GC if NSAID and colchicine are contraindicated, as in individuals with CKD or endstage renal disease (ESRD), or in the elderly ${ }^{5}$. However, GC should be used with caution in individuals with endstage or refractory heart failure, or diabetes with poor glycemic control.

In these medically complex individuals, the interleukin (IL)-1 receptor antagonist, anakinra, may be considered. Anakinra blocks the downstream effects of the NLRP3 (NOD, LRR, and pyrin domain-containing 3) inflammasome. This protein complex is activated by both MSU and CPP crystals, resulting in the caspase- 1 cleavage of pro-IL- $1 \beta$ to active $\mathrm{IL}-1 \beta$, an inflammatory cytokine ${ }^{6,7}$. The standard anakinra dosing is derived from the pilot study by So, et al, in which individuals with acute gouty arthritis were given anakinra $100 \mathrm{mg}$ subcutaneously daily for $3 \mathrm{doses}^{8}$. Several observational studies, including one from our center, have demonstrated the rapid efficacy of anakinra on pain and swelling in acute gout attacks ${ }^{9-18}$. The observational data for

Personal non-commercial use only. The Journal of Rheumatology Copyright @ 2019 . All rights reserved. 
CPP crystal arthritis have been more limited, but anakinra has also been shown to be effective for acute episodes as well as maintenance therapy $14,19,20,21,22,23,24$.

In the current single-center, retrospective, observational study, we assessed the efficacy and safety of anakinra in medically complex, hospitalized patients with acute gout and CPP crystal arthritis. The 26 patients reported in our prior publication were not included in the current study.

\section{MATERIALS AND METHODS}

Adult patients hospitalized at the University of Washington Medical Center (UWMC) and Harborview Medical Center (HMC) and who were treated with anakinra during their admission were identified for inclusion through the Leaf application, the interface for the University of Washington's deidentified clinical data repository. Information was available for admissions from January 2014 to the date of the analysis, December 10,2017. A total of 120 individual patients were identified. We conducted a chart review and excluded patients who received anakinra for indications other than gout or CPP crystal arthritis; the remaining 100 patient charts were reviewed for basic demographic information, comorbidities, laboratory data, pain scores, joint involvement, prior treatment, anakinra dosing and frequency, response to anakinra treatment, concurrent infections and surgical interventions, and adverse effects of anakinra administration. The diagnoses of gout and CPP crystal arthritis were defined as follows: by joint aspiration yielding MSU or CPP crystals; and/or clinical diagnosis by a rheumatologist; and/or acute joint pain and swelling in the setting of prior gout or CPP crystal arthritis diagnosis, with alternative diagnoses excluded. At UWMC and HMC, the administration of anakinra was not restricted to the rheumatology consultation service.

Infections and surgical interventions were considered concurrent if they occurred during the index hospitalization within a month of anakinra treatment. Infections were considered concurrent and unrelated to anakinra administration unless they were attributed as such in the discharge summary.

Response to anakinra treatment was determined from chart review of inpatient provider documentation, as well as recorded pain scores $(0-10$ on a numeric scale). Responses were rated on a scale of no response, partial response, significant response, and complete resolution. Partial response was denoted by documented clinical response by the clinician, such as commentary in the chart that the patient had "modest," "some," or "mild" improvement; or situations in which 1 joint was improved but another was not. Significant response was denoted by any combination of the following: functional improvement, such as ability to walk or bear weight on the affected joint; movement or range of motion of the affected joint with minimal pain; improved swollen or tender joints on examination; or documented clinical response such as "dramatic" or "significant" improvement. If there was no documentation of the above, then a change in the average daily pain score by at least 2 points was considered a significant response ${ }^{25}$. Complete resolution was denoted by chart documentation stating that the patient's flare was completely resolved. Cases of incomplete documentation were denoted as "NA."

Our study (STUDY00003614) was approved by the institutional review board of the University of Washington Human Subjects Division.

\section{RESULTS}

We identified 100 individuals hospitalized at UWMC or HMC between January 2014 and December 2017, who received anakinra for the acute treatment of gout or CPP crystal arthritis while inpatients. These individuals accounted for a total of 115 separate episodes of arthritis. Baseline data are shown in Table 1 . The population was $82 \%$ male, with an average age of 60 years. The majority were white (64\%);
Table 1. Baseline demographics.

\begin{tabular}{lc}
\hline Variables & Values \\
\hline Age, yrs & $60 \pm 15$ \\
Sex, male & 82 \\
Race/ethnicity & \\
$\quad$ White & 64 \\
$\quad$ Black & 19 \\
$\quad$ Asian or Pacific Islander & 15 \\
$\quad$ Native American & 1 \\
$\quad$ Unknown & 1 \\
Comorbidities & \\
$\quad$ Chronic kidney disease or ESRD & 45 \\
$\quad$ Heart failure & 43 \\
$\quad$ Diabetes mellitus & 27 \\
Chronic anticoagulation & 22 \\
$\quad$ History of transplantation & 14 \\
Infection within the same admission, no. episodes & 34 \\
Operation within the same admission, no. episodes & 29 \\
Serum uric acid, mg/dla & \\
Serum creatinine, mg/dl & \\
Pain score (0-10) & \\
Duration of gout flare, days $_{\text {Indication for anakinra }}$ & $8.6 \pm 4.1$ \\
$\quad$ Comorbidities & $1.9 \pm 1.9$ \\
Failure of other therapies & $4.1 \pm 2.8$ \\
Reason not documented & $4.8 \pm 5.3$ \\
& \\
&
\end{tabular}

Values are mean \pm SD or \% unless otherwise specified. a Serum uric acid obtained most proximately to episode of arthritis. ${ }^{b}$ Serum creatinine obtained most proximately to the administration of anakinra; ESRD patients not included. ${ }^{c}$ Average of pain scores on a numeric pain scale $(0-10)$ obtained at initiation of anakinra. ${ }^{\mathrm{d}}$ Indications for anakinra administration could be multiple and overlapping within the same individual. ESRD: endstage renal disease.

$19 \%$ were black, $15 \%$ Asian or Pacific Islander, $1 \%$ Native American, and $1 \%$ did not report a race or ethnicity. Comorbidities included heart failure in $43 \%$, CKD or ESRD in $45 \%$, and diabetes mellitus in $27 \%$. In addition, chronic anticoagulation was used by $22 \%$, and $14 \%$ had a history of organ transplantation requiring antirejection immunosuppression. Fifty-eight percent of individuals had 2 or more of these comorbid conditions. The average serum creatinine measured proximately to the episode of arthritis was 1.9 $\mathrm{mg} / \mathrm{dl}$.

Ninety-three of the patients had gout, of which 22 were crystal-proven during the index admission. Of the 7 patients who had CPP crystal arthritis, 5 were crystal-proven. Of the remaining patients who did not have a confirmatory synovial fluid analysis during their index admission, about $10 \%$ reported a prior crystal-proven diagnosis of gout. The average serum uric acid level at the time of the flare was $8.6 \mathrm{mg} / \mathrm{dl}$ and was available for 90 episodes. Patients had an average pain score of 4.1 on a numeric pain scale (range $0-10$ ).

Monoarthritis was seen in 43 episodes, oligoarthritis in 56 , and polyarthritis in 15 ; one episode presented as a systemic inflammatory response alone in the absence of joint manifestations. The knee was involved in 37 episodes, the

Personal non-commercial use only. The Journal of Rheumatology Copyright $\odot$ (2019. All rights reserved 
ankle in 35 , the first metatarsophalangeal joint in 25 , the wrist in 25, the elbow in 22, and the small joints of the hand in 21. The back and shoulder were involved in 1 episode each (data not shown).

Concurrent infection was present in 34 arthritis episodes. This included cellulitis or abscess in 7, bacteremia in 6 ( 3 with Staphylococcus, 1 with Pseudomonas, 2 with Klebsiella), septic arthritis in 6, urinary tract infection in 5, Clostridium difficile colitis in 5, pneumonia in 3 , cytomegalovirus viremia in 1 , and infectious endocarditis in 1.

Recent surgical intervention was found associated with 29 arthritis episodes. This included articular washout in 5, incision and drainage or debridement in 4, other orthopedic procedures in 4 , cardiac procedures in 4 (either a valvular surgery or placement of a left ventricular assist device), transplantation in 3 ( 2 orthotopic heart and 1 orthotopic liver transplantations), video-assisted thoracic surgery in 3, endovascular procedures in 2, neurosurgical procedure in 1 , and 3 procedures classified as other.

Other treatments for the acute arthritis (Table 2) included NSAID for 2 episodes ( 1 day of treatment in 1 case; $>2$ days in the other case); colchicine for 26 episodes ( $\leq 2$ doses in 19 ; $>2$ doses in 7 ); oral prednisone of $\geq 10 \mathrm{mg}$ daily in 23 episodes ( 1 received 1 day of treatment; the rest received $\geq 2$ days of treatment); intraarticular GC injection for 7 episodes; and operative washout in 3 episodes. No other treatment apart from anakinra was given for 55 episodes.

The decision to treat with anakinra was due to the patient's underlying comorbidities in $84 \%$ of episodes, and due to failure of other therapies in $48 \%$ of episodes; some patients had both indications for the use of anakinra. The average duration of the arthritis episode prior to receiving anakinra was 4.8 days. Various dosing regimens of anakinra were used (Table 3). Twenty-four episodes were treated with a single dose; $100 \mathrm{mg}$ daily for 2 doses was given for 13 episodes; $100 \mathrm{mg}$ daily for 3 doses was given for 52 episodes; $100 \mathrm{mg}$ daily for $>3$ doses was given for 8 episodes; $100 \mathrm{mg}$ every other day (renally dosed) for 2 doses was given for 4

Table 2. Prior treatments, by episode.

\begin{tabular}{lc}
\hline Treatments & Episodes, $\mathrm{n}$ \\
\hline NSAID & \\
$\quad 1$ day & 1 \\
$\quad>1$ day & 1 \\
Colchicine & \\
$\quad \leq 2$ doses & 19 \\
$\quad>2$ doses & 7 \\
Prednisone & \\
$\quad 1$ day & 1 \\
$\quad>1$ day & 22 \\
Intraarticular glucocorticoid & 7 \\
Operative washout & 3 \\
No other therapy & 55
\end{tabular}

NSAID: nonsteroidal antiinflammatory drug.
Table 3. Anakinra dosing regimens, by episode

\begin{tabular}{lc}
\hline Anakinra Doses & Episodes, $\mathrm{n}$ \\
\hline $100 \mathrm{mg}$, once & 24 \\
$100 \mathrm{mg}$ daily & 13 \\
2 doses & 52 \\
3 doses & 8 \\
$>3$ doses & \\
100 mg, every other day & 4 \\
2 doses & 13 \\
3 doses & 1 \\
$>3$ doses & \\
\hline
\end{tabular}

episodes; $100 \mathrm{mg}$ every other day for 3 doses was given for 13 episodes; and $100 \mathrm{mg}$ every other day for $>3$ doses was given for 1 episode.

Response to anakinra is shown in Table 4. Eighty-six episodes of arthritis had partial or complete response to anakinra within 4 days of the first dose. Sixty-six episodes had partial or complete response within 1 day of administration of the first dose. There was only a partial response in 7 episodes and no response in 6 . There was insufficient information to determine the response in 14 episodes. In 36 episodes, patients were discharged from the hospital within 4 days of receiving the first dose of anakinra.

Overall, anakinra was well tolerated. Two individuals had leukopenia attributed to anakinra administration (1 new, 1 with worsening of preexisting white blood cell counts). Worsening of bicytopenia, injection site reaction, and nausea occurred in 1 individual each.

\section{DISCUSSION}

To our knowledge, this is the largest observational study of anakinra use in the inpatient setting for the acute treatment of crystal-associated arthritis. Our findings build upon our earlier study, in which 26 individuals were treated for a total of 40 episodes with few adverse events ${ }^{10}$. In our current study, we observed a rapid response to anakinra, with $75 \%$ of episodes significantly improving or completely resolving within 4 days of the first dose, and $57 \%$ of episodes within 1 day of the first dose. These data also support the use of this biologic agent in those with active or recent infections, those who are peri- or postoperative, and in those immunosuppressed for transplant rejection purposes.

The first-line agents for the treatment of acute gout or CPP

Table 4. Responses by episode.

Responses $\quad$ Episodes, $\mathrm{n}$

Significant response or complete resolution by 4 days $\quad 86$

Delayed response $>4$ days 2

Partial response $\quad 7$

Nonresponse 6

Insufficient information $\quad 14$ 
crystal arthritis are NSAID, colchicine, and GC. These are agents that may fail or be poorly tolerated in a medically complex patient population with multiple comorbidities that include diabetes, CKD, and congestive heart failure. Prior observational studies of anakinra have shown its efficacy in this population. Other IL-1 inhibitors, canakinumab and rilonacept, have been shown in trials to be effective for the treatment of acute and chronic gout, as well as for flare prophylaxis while initiating urate-lowering therapy $26,27,28$.

Forty-five percent of the patients in our study had CKD or ESRD. In most of these cases, anakinra was dose-reduced to $100 \mathrm{mg}$ every other day, as described in prior studies ${ }^{29}$. We observed the safety of anakinra in these patients with renal dysfunction, complementing the findings of Loustau, et al in their retrospective study of 25 patients with $\mathrm{CKD}^{30}$.

With regard to safety, our study identified 29 episodes in which individuals received anakinra in the setting of concurrent infection. These infections ranged from localized infections such as cellulitis to systemic infections such as bacteremia. In many of these cases, the decision to initiate anakinra was made following multidisciplinary discussions with the infectious disease consultants regarding the risks and benefits. All individuals received appropriate antibiotic coverage. There was no indication of worsening of infection in these patients that could be attributed to the anakinra administration. This finding is in agreement with other observational studies that included individuals with active infection $^{10,12,13,15}$.

Twenty-six episodes of anakinra administration were in the setting of recent surgical intervention, without evidence for subsequent surgical complications. Our study also identified 14 patients with a history of solid organ or stem cell/bone marrow transplantation, including 3 who had transplantations during their index hospitalizations. No complications were attributed to the administration of anakinra. Prior published cases of transplant recipients receiving anakinra for crystal-associated arthritis include 10 individuals with renal transplants ${ }^{15,30,31}$ and 2 with orthotopic liver transplants ${ }^{15}$. The safety of anakinra in a bone marrow transplant recipient has been described in a case report of familial Mediterranean fever ${ }^{32}$. We add to these reports 2 individuals who received heart transplants during the same admission that they were given anakinra.

Although we are unable to determine the effect of anakinra administration on the length of stay, we did note that in $31 \%$ of episodes, patients were discharged from the hospital within 4 days of the first anakinra dose. We could extrapolate that these patients experienced responses significant enough to allow them to leave the hospital. Given the rapid efficacy of anakinra, this treatment may be more cost-effective in those who are refractory to other less costly treatments. Our findings are supported by a case-control study by Singh, et $a l$, in which 16 patients receiving anakinra for acute crystal-associated arthritis, who were matched with 32 controls receiving conventional therapy, were found to have a length of stay that was 2 days shorter, on average ${ }^{33}$. The partial or nonresponse to anakinra seen in some of our patients may be explained by the short half-life of anakinra (4-8 h), other involved cytokines, or by patient selection as discussed below.

This study has its strengths in the number of patients included, and the high prevalence of comorbidities. However, as a retrospective observational study, it has limitations. About half of the patients did not have a crystal-proven diagnosis; a noncrystal-associated arthritis may have been misclassified as gout or CPP crystal arthritis, thus accounting for poor or inadequate anakinra response as discussed. Our previous observational study's ${ }^{10}$ response rates were $67 \%$ of patients had a 1-day significant response rate and $90 \%$ achieved a significant response within 4 days (compared to $57 \%$ and $75 \%$, respectively, in the current study). Since the first study was published, the efficacy of anakinra for gouty arthritis is widely recognized within our institution and anakinra therapy can now be instituted by the primary inpatient team without rheumatology involvement. In 20 cases of anakinra administration, the order was initiated prior to, or without the involvement of, the rheumatology consultation service. This may affect the number of patients who actually have crystal-associated arthritis because few were crystal-proven. In addition, there was no control population to serve as a comparison for the efficacy of anakinra therapy. These patients had significant comorbidities that limited their use of other pharmacological therapies to treat their acute arthritis; thus, they cannot easily be compared to a less medically complex population that did not receive anakinra. Because there were no formal standards of documentation of improvement of arthritis flares, the response criteria we used cannot account for inaccuracies of over- or underestimating response. We cannot account for all possible adverse events attributable to the anakinra, because these may not have been recorded or documented in the discharge summaries. Further adverse events may have occurred following the hospitalization, which may not have been documented in the electronic medical record.

Controlled trials are required to solidify the efficacy of anakinra for use as an acute therapy for gout or CPP crystal arthritis, particularly in medically complex inpatients who may have concurrent infections or recent surgical procedures.

Acute attacks of gout or CPP crystal arthritis can be highly symptomatic and can cause frequent complications in inpatient admissions, particularly in a medically complex patient population for whom standard therapies are contraindicated, poorly tolerated, or unsuccessful. Anakinra should be considered in these patients, because it has a rapid onset of action and is well tolerated. Our large retrospective study supports its use in hospitalized patients, including those with concurrent infections, transplant recipients, or those who have undergone recent surgical interventions.

Personal non-commercial use only. The Journal of Rheumatology Copyright $\odot$ 2019. All rights reserved 


\section{ACKNOWLEDGMENT}

The authors acknowledge the University of Washington Division of Rheumatology for its thoughtful comments and critique of this study during the Works in Progress presentations, and Dr. Julianna Desmarais (Division of Arthritis and Rheumatic Diseases, Department of Medicine, Oregon Health \& Science University, Portland, Oregon, USA) for her insights regarding a similar project undertaken at her institution.

\section{REFERENCES}

1. Zhu Y, Pandya BJ, Choi HK. Prevalence of gout and hyperuricemia in the US general population: the National Health and Nutrition Examination Survey 2007-2008. Arthritis Rheum 2011;63:3136-41.

2. Zhu Y, Pandya BJ, Choi HK. Comorbidities of gout and hyperuricemia in the US general population: NHANES 2007-2008. Am J Med 2012;125:679-87.

3. Singh JA, Sarkin A, Shieh M, Khanna D, Terkeltaub R, Lee SJ, et al. Health care utilization in patients with gout. Semin Arthritis Rheum 2011;40:501-11.

4. Singh JA, Strand V. Gout is associated with more comorbidities, poorer health-related quality of life and higher healthcare utilisation in US veterans. Ann Rheum Dis 2008;67:1310-6.

5. Khanna D, Khanna PP, Fitzgerald JD, Singh MK, Bae S, Neogi T, et al; American College of Rheumatology. 2012 American College of Rheumatology guidelines for management of gout. Part 2: therapy and antiinflammatory prophylaxis of acute gouty arthritis. Arthritis Care Res 2012;64:1447-61

6. Martinon F, Pétrilli V, Mayor A, Tardivel A, Tschopp J. Gout-associated uric acid crystals activate the NALP3 inflammasome. Nature 2006;440:237-41.

7. So A, Ives A, Joosten LA, Busso N. Targeting inflammasomes in rheumatic diseases. Nature Rev Rheumatol 2013;9:391-9.

8. So A, De Smedt T, Revaz S, Tschopp J. A pilot study of IL-1 inhibition by anakinra in acute gout. Arthritis Res Ther 2007;9:R28.

9. Chen K, Fields T, Mancuso CA, Bass AR, Vasanth L. Anakinra's efficacy is variable in refractory gout: report of ten cases. Semin Arthritis Rheum 2010;40:210-4.

10. Ghosh P, Cho M, Rawat G, Simkin PA, Gardner GC. Treatment of acute gouty arthritis in complex hospitalized patients with anakinra. Arthritis Res Care 2013;65:1381-4.

11. Ottaviani S, Moltó A, Ea HK, Neveu S, Gill G, Brunier L, et al. Efficacy of anakinra in gouty arthritis: a retrospective study of 40 cases. Arthritis Res Ther 2013;15:R123.

12. Thueringer JT, Doll NK, Gertner E. Anakinra for the treatment of acute severe gout in critically ill patients. Semin Arthritis Rheum 2015;45:81-5.

13. Vitale A, Cantarini L, Rigante D, Bardelli M, Galeazzi M. Anakinra treatment in patients with gout and type 2 diabetes. Clin Rheumatol 2015;34:981-4.

14. Aouba A, Deshayes S, Frenzel L, Decottignies A, Pressiat C, Bienvenu B, et al. Efficacy of anakinra for various types of crystal-induced arthritis in complex hospitalized patients: a case series and review of the literature. Mediators Inflamm 2015;2015:792173.

15. Palma C, Topping T, Tabechian D. Anakinra is effective and well tolerated in medically complex patients including transplant recipients with gout [abstract]. Arthritis Rheumatol 2016;68 Suppl 10:215

16. Petite SE. Effectiveness of anakinra in acute gout: a retrospective review of initial and refractory therapy. Am J Ther 2017;24:e633-4.

17. Sharma E, Terkeltaub R. A case control study of anakinra use for acute gout in a VA patient cohort reveals association with East Asian descent, high urate burden, and increased co-morbidities and all-cause mortality [abstract]. Arthritis Rheumatol 2017;69 Suppl 10:1142.
18. Desmarais J, Chu CQ. Utility of anakinra in acute crystalline diseases: A retrospective study comparing a university hospital with Veterans Affairs Medical Center [abstract]. Arthritis Rheumatol 2017;69 Suppl 10:2090.

19. McGonagle D, Tan AL, Madden J, Emery P, McDermott MF. Successful treatment of resistant pseudogout with anakinra. Arthritis Rheum 2008;58:631-3.

20. Announ N, Palmer G, Guerne PA, Gabay C. Anakinra is a possible alternative in the treatment and prevention of acute attacks of pseudogout in end-stage renal failure. Joint Bone Spine 2009;76:424-6.

21. Couderc M, Mathieu S, Glace B, Soubrier M. Efficacy of anakinra in articular chondrocalcinosis: report of three cases. Joint Bone Spine 2012;79:330-1

22. Moltó A, Ea HK, Richette P, Bardin T, Lioté F. Efficacy of anakinra for refractory acute calcium pyrophosphate crystal arthritis. Joint Bone Spine 2012;79:621-3.

23. Ottaviani S, Brunier L, Sibilia J, Maurier F, Ardizzone M, Wendling $\mathrm{D}$, et al. Efficacy of anakinra in calcium pyrophosphate crystal-induced arthritis: a report of 16 cases and review of the literature. Joint Bone Spine 2013;80:178-82.

24. Thomas M, Forien M, Palazzo E, Dieude P, Ottaviani S. Efficacy and tolerance of anakinra in acute calcium pyrophosphate crystal arthritis: a retrospective study of 33 cases. Clin Rheumatol 2019;38:425-30.

25. Salaffi F, Stancati A, Silvestri CA, Ciapetti A, Grassi W. Minimal clinically important changes in chronic musculoskeletal pain intensity measured on a numerical rating scale. Eur J Pain 2004;8:283-91.

26. So A, De Meulemeester M, Pikhlak A, Yücel AE, Richard D, Murphy V, et al. Canakinumab for the treatment of acute flares in difficult-to-treat gouty arthritis: Results of a multicenter, phase II, dose-ranging study. Arthritis Rheum 2010;62:3064-76.

27. Terkeltaub RA, Schumacher HR, Carter JD, Baraf HS, Evans RR, Wang J, et al. Rilonacept in the treatment of acute gouty arthritis: a randomized, controlled clinical trial using indomethacin as the active comparator. Arthritis Res Ther 2013;15:R25.

28. Terkeltaub R, Sundy JS, Schumacher HR, Murphy F, Bookbinder S, Biedermann $\mathrm{S}$, et al. The interleukin 1 inhibitor rilonacept in treatment of chronic gouty arthritis: results of a placebo-controlled, monosequence crossover, non-randomised, single-blind pilot study. Ann Rheum Dis 2009;68:1613-7.

29. Yang BB, Baughman S, Sullivan JT. Pharmacokinetics of anakinra in subjects with different levels of renal function. Clin Pharmacol Ther 2003;74:85-94.

30. Loustau C, Rosine N, Forien M, Ottaviani S, Juge PA, Lioté F, et al Effectiveness and safety of anakinra in gout patients with stage 4-5 chronic kidney disease or kidney transplantation: a multicentre, retrospective study. Joint Bone Spine 2018; 85:755-60.

31. Mulders-Manders CM, Baas MC, Molenaar FM, Simon A. Peri- and postoperative treatment with the interleukin-1 receptor antagonist anakinra is safe in patients undergoing renal transplantation: Case series and review of the literature. Front Pharmacol 2017;8:342.

32. Petropoulou AD, Robin M, Socié G, Galicier L. Transmission of familial Mediterranean fever mutation after bone marrow transplantation and successful treatment with anakinra. Transplantation 2010;90:102-3.

33. Singh S, Ocon A, Mehta V, Musa S, Peredo R. Anakinra is more effective at reducing pain from acute crystal induced arthritis when compared to conventional therapy: A retrospective review at a tertiary care center [abstract]. Arthritis Rheumatol 2018;70 Suppl 10:1295.

$$
\text { Personal non-commercial use only. The Journal of Rheumatology Copyright (C) 2019. All rights reserved. }
$$

